\title{
BREVE DISCUSIÓN DE LA ECONOMÍA POLÍTICA DE LOS FLUJOS FINANCIEROS ILÍCITOS EN EL MUNDO Y AMÉRICA LATINA
}

\section{BRIEF DISCUSSION OF THE ECONOMICAL POLICY OF ILLICIT FINANCIAL FLOWS AROUND THE WORLD AND LATIN AMERICA}

KATIUSKA KING MANTILLA ${ }^{1}$

Recibido: 12 de abril

Aceptado: 1 de mayo

${ }_{1}^{1}$ Msc. Universidad de Sevilla. Universidad Central del Ecuador. 



\section{BREVE DISCUSIÓN DE LA ECONOMÍA POLÍTICA DE LOS FLUJOS FINANCIEROS ILÍCITOS EN EL MUNDO Y AMÉRICA LATINA $^{1}$}

\section{BRIEF DISCUSSION OF THE ECONOMICAL POLICY OF ILLICIT FINANCIAL FLOWS AROUND THE WORLD AND LATIN AMERICA}

Katiuska King Mantilla

Palabras clave: flujos financieros ilícitos, acuerdos institucionales internacionales, economía política internacional, regulación financiera

Keywords: illicit financial flows; international institutional arrangements, international political economy, financial regulation

Clasificación JEL: F55, G18, P16.

\section{RESUMEN}

El presente artículo analiza si la una perspectiva global o regional, idenproblemática de los flujos financieros tificando puntos de encuentro y desenilícitos (FFI) puede ser explicada desde cuentro entre los intereses de los países

\footnotetext{
1 Publicado en la Revista Economía Vol. 70(111), 201-208. Recuperado a partir de http://revistadigital.uce. edu.ec/index.php/ECONOMIA/article/view/1397
} 
en función de las repercusiones que esta genera, así como de las propuestas de solución en actual discusión. El dumping social y la estabilidad financiera se cuentan entre los principales elementos de encuentro mientras que el reciclaje financiero y la representación de las instituciones que discuten las soluciones, se pueden considerar como componentes de desencuentro fundamentales.

\section{ABSTRACT}

The present article analyzes if the problem of the illicit financial flows (IFF) can be explained from a global or regional perspective, identifying points of agreement and disagreement among the interests of the countries depending on the effects that it generates, as well as of the current proposals of solution. The social dumping and the financial stability are told in the principal elements of agreement whereas the financial recycling and the institutions that discuss the solutions are key components of disagreement.

\section{INTRODUCCIÓN}

El término "flujos financieros ilícitos" fue acuñado por organizaciones de la sociedad civil que promueven la transparencia financiera para exponer una problemática palpitante y clave para la economía y la sociedad, tanto a nivel macro y microeconómico como de la economía política internacional. El término "ilícito" no hace solo referencia a la economía explícitamente delictiva como la corrupción, la trata de personas y el narcotráfico, cuyos recursos una vez blanqueados pueden buscar refugio externo, sino también a prácticas para evadir o eludir impuestos, sobre todo, por parte de empresas multinacionales (EMN) y personas de alto patrimonio.

Los flujos financieros ilícitos son "movimientos transfronterizos de dinero que han sido ilegalmente obtenidos, transferidos o usados" (Tax Justice Network, 2015). Estos movimientos se realizan tomando varios senderos de países hasta llegar a los circuitos financieros mundiales en los que se cuentan principalmente Nueva York y Londres, y de importancia para los FFI, Holanda, Suiza, Singapur e Irlanda (García-Bernardo, Fichtner, Takes, \& Heemskerk, 2017). Es decir, los FFI no permanecen guardados 
en los países llamados paraísos fiscales, sino que se reciclan a través de los centros financieros y luego pueden regresar en forma de deuda o nuevas inversiones.

El capitalismo contemporáneo mantiene sus riesgos y contradicciones, más aún en el contexto de una globalización económica y financiera marcada por la actividad de esta última, en la que se observa con claridad la concepción del capital, planteada por Marx, como relaciones sociales y de poder.

Para ello se debe partir de que actualmente se considera a la economía sin aparente influencia de la política lo que la posiciona como neutra, empezando por los temas que se investigan. Bhaduri (2011) nos recuerda que la economía política debe ayudar a entender mejor el mundo en el que vivimos antes que inventar abstracciones de la realidad, alejadas de la complejidad social y económica. Lo que sucede hoy por hoy en la investigación económica es que se ajustan las técnicas a las temáticas en vez de buscar temas relevantes que expliquen la situación existente y que cuestionen el sentido común económico dominante.

Este artículo analiza desde la economía política si los FFI deberían ser considerados desde lo global o desde los países periféricos; para la región latinoamericana algunas aristas de este fenómeno han sido analizadas (King, 2018). La metodología utilizada en este artículo parte de examinar los distintos ángulos de los FFl, identificando puntos de encuentro y desencuentro entre grupos de países que se benefician o perjudican de ellos.

Este artículo introduce la temática y continúa con un breve recuento teórico de economía política internacional; discute los elementos más relevantes de los FFI en clave de economía política internacional y concluye.

\section{ECONOMÍA POLÍTICA INTERNACIONAL}

La economía política internacional se tiende a asociar solo con el comercio internacional (Lake, 2008, p. 758), cuando también incluye a la política expresada en intereses y relaciones de poder así como a instituciones, autoridades y mercados.
Las categorías clásicas de economía política internacional son el realismo, el marxismo y el liberalismo. Mientras que las actuales categorías tienen explicaciones desde una mirada meramente internacional a otras que provienen de realidades domésticas, así como las que 
se basan en las instituciones o en la sociedad (Frieden \& Lake, 2000).

En la perspectiva marxista, destaca la visión de Lenin (1939) sobre la fusión de capital bancario con el capital industrial y la consecuente aparición de una oligarquía financiera en pocos bancos. Esta oligarquía permitió la exportación de capital para obtener mejores retornos y aprovechar las ventajas de países menos avanzados en ganancias precio de la tierra, salarios y materias primas (Ibid., p. 99).

La teoría de la dependencia estudiada por Prebisch identifica el deterioro de los términos de intercambio y establece como categorías de análisis a los países periféricos y a los países centrales planteando que la periferia transfiere valor al centro. Luego en los ochenta, Prebisch (1987) añade que al interior de América Latina algunos países exportaron bienes manufacturados a los países de la región manteniendo el esquema centro-periferia en otra escala.

Gilpin (2001) recalca la interacción de temas económicos y políticos e identifica las relaciones económicas políticas transnacionales y el poder de las EMN como nuevos actores que dominan la escena internacional.
Otra perspectiva de análisis es la de Susan Strange que integra la política, las instituciones, la autoridad y los mercados. Para ella, la teoría de las relaciones internaciones con el afán de no incluir la tradición marxista dejó por fuera la economía política global. Strange (1994, pp. 45-138) plantea que existen cuatro canales a través de los cuales se ejerce el poder estructural en las relaciones internacionales: la seguridad, el conocimiento, la producción y las finanzas, los tres últimos relacionados con los FFI y la inversión extranjera directa (IED). La seguridad indirectamente está vinculada con los FFI a través de venta ilegal de armamento. Strange (2002) sostiene que es el poder estructural menos visible detrás de la agenda el que debe ser examinado para un análisis significativo de la economía política internacional.

Esta perspectiva teórica permite estudiar los FFI y a las finanzas como instrumento canalizador de estos flujos en los mercados internacionales. La existencia de los FFI introduce mayor complejidad a lo que Strange (1986) denominó "Capitalismo de casino". A continuación, se discute en clave de economía política internacional el ámbito de los FFI. 


\section{DISCUSIÓN}

Este artículo se pregunta en qué medida los FFI son un fenómeno global o regional y propone puntos principales de encuentro y desencuentro.

\section{Puntos de desencuentro}

\section{- Definición de los FFI}

El primer punto se refiere a la definición de los FFl, sobre la cual no existe consenso por cuanto se considera a la elusión de impuestos como algo permitido por las normativas de los países y que, por tanto, entra dentro de una zona gris. En términos semánticos, según la Real Academia de la Lengua (RAE), lo ilegal es contrario a la Ley mientras que lo ilícito es lo "no permitido legal o moralmente". Así, lo ilegal infringe una norma específica mientras que lo ilícito no está permitido pero los marcos normativos no lo prevén y por tanto no lo sancionan.

En el campo tributario, existen especialistas de las principales auditoras mundiales dedicados a buscar vacíos legales con el objetivo de reducir las cargas impositivas. Más allá de caracterizar moralmente estos actos como una ganancia ilegítima fruto del no pago de tributos, así como de la intencionalidad dolosa del uso de huecos administrativos, la clave está en que estos vacíos pueden ser aprovechados por quienes pueden pagar por estos servicios: grandes EMN a diferencia de las pequeñas y medianas empresas domésticas. Por tanto, una definición estrecha de los FFI interesa a los países donde se alojan estas multinacionales. En los países periféricos, sobre todo latinoamericanos, la institucionalidad en materia tributaria es débil, con pocas excepciones y la creación de nuevas normas avanza de forma lenta fruto de influencias y presiones políticas. Para los países periféricos interesa una definición amplia de los FFI que incluya la elusión como parte de lo ilícito por cuanto estas prácticas erosionan sus bases imponibles.

\section{- Inversión extranjera directa (IED)}

Para recibir IED los países periféricos compiten entre ellos con toda suerte de incentivos que representan sacrificios tributarios, así como instrumentos legales de protección para las EMN. En la región latinoamericana existe una mirada de realismo mágico a propósito de la IED ya que no se observan sus efectos reales en la balanza de pagos.

Esta es volátil y está atravesada por temas de FFI que la hacen contribuir cada vez menos a la formación bruta de capital fijo (FBKF) y más al pago de regalías (King, 2018). Es importante mencionar que lo que beneficia a las 
EMN en términos de acuerdos con territorios opacos también lo hace al lavado de dinero.

\section{- Prácticas tributarias agresivas e instituciones de debate}

Q. Hong \& Smart (2010) afirman que la planificación tributaria beneficia a los países desarrollados. Estas prácticas tributarias agresivas están definidas por el proyecto Erosión de la Base imponibley traslado de los beneficios conocido como BEPS, por sus siglas en inglés². Y este es el segundo punto de desencuentro dado que, este proyecto lo impulsa la OECD donde evidentemente existen intereses discordantes.

El objetivo principal de este proyecto de agrupar las cuentas de las EMN no se cumplió y se mantiene el tratamiento de cada filial como un ente separado, es decir las cuentas no se consolidan.

La OECD mantiene un Centro de Política y Administración Tributaria que para el año 2013 contaba un presupuesto de 10 millones de euros. El grupo de países G20 y G7 tienen influencia en la definición de la agenda y la toma de decisiones se restringe a los países miembros de la OECD. Una institución en la cual los países periféricos estarían mejor representados es el Comité Tributario que pertenece al Consejo Económico y Social de Naciones Unidas (ECOSOC) que por su parte mantuvo para el 2013 un presupuesto de solo 175 mil dólares y un staff de 2,5 personas a tiempo completo (King, 2016). Los países ricos tienen interés en mantener las decisiones en la OECD.

\section{- Posibles mecanismos de solución}

La compleja estructura societaria de filiales facilita el arbitraje tributario ya que las EMN no consolidan sus cuentas y mantienen información por cada filial. Una de las soluciones propuestas a las prácticas abusivas de las EMN es que presenten un reporte consolidado país por país (country by country report) de sus actividades económicas para conocer su situación de forma integral y proponer un reparto más equitativo de los impuestos que dejan de pagar.

Con esta información, la Comisión independiente para la reforma de la tributación internacional corporativa (ICRICT) busca proponer una imposición unitaria ${ }^{3}$ con un reparto más equitativo de los impuestos que dejan de pagar las EMN.

\footnotetext{
2 Este proyecto pretendía un cambio en las reglas impositivas internacionales para asegurar que las multinacionales paguen sus impuestos en el lugar donde las actividades se realizan y se crea valor agregado. Terminó generando 15 acciones con sus respectivas recomendaciones, algunas difíciles de implementar. ${ }^{3}$ En inglés "unitary taxation"y las siglas del ICRICT corresponden a Independent Commission for the reform of
} 
Sin embargo, cuando se agrupen las cuentas de las EMN habrá interés de los países donde estas residen por recibir la mayor parte de los impuestos omitidos. Si bien el reporte país por país es necesario y puede ser considerado un punto de encuentro, la forma como se distribuyan los recursos y nuevamente la institución que tome la batuta en esta definición será un punto de desencuentro. Esto se podría anticipar con una propuesta propia de distribución de los impuestos omitidos.

\section{- Reciclaje financiero y facilitadores}

Además de los impuestos, es importante anotar que el dinero de los FFI se recicla en instrumentos financieros de los países ricos. En términos de financiamiento para el desarrollo, en los países periféricos nos contentamos con tener un acceso cíclico —es decir, fluido en épocas de bonanza - a los mercados internacionales para la colocación de bonos, condicionado por la percepción del riesgo de las calificadoras.

Este reciclaje financiero en el norte no es bien entendido cuando se culpabiliza a ciertos países periféricos considerados como paraísos fiscales o facilitadores ya que el dinero no se queda ahí sino en los países ricos. Por tanto, la discusión de los paraísos fiscales deja de hablar de los países ricos a donde sí llega el dinero y es, por ello, un punto de desencuentro.

\section{Puntos de encuentro}

\section{- Importancia de los tributos en la política económica}

Las esferas que globalmente nos acercan a la problemática de los FFI son los efectos que la elusión y evasión de impuestos generan en todos los países. Los temas impositivos son relevantes para el financiamiento propio de los países tanto en la política fiscal como en la social. Actualmente, se posiciona un discurso a favor del mercado, contra los impuestos y el Estado que no facilita reformas tributarias progresivas.

El cuestionamiento a los impuestos tiene implicaciones globales, tanto para los estados de bienestar ya consolidados en el norte como para los países del sur que adolecen los efectos de la desigualdad, sobre todo los latinoamericanos. Las elites económicas mundiales, propietarias de conglomerados empresariales no ven "necesaria" esta solidaridad y la cuestionan ya que no requieren servicios sociales y prefieren aumentar sus beneficios empresariales y personales. 


\section{- Dumping social}

Un factor que las EMN buscan al analizar sus nuevas localizaciones es el pago de salarios más bajos. Este punto perjudica a todos los países por cuanto precariza el trabajo en los países periféricos y genera desempleo en los países ricos. Las fusiones y adquisiciones que realizan las EMN sirven para utilizar capacidades productivas instaladas previamente por otras empresas en países con menores costos productivos. El dumping social se convierte en un factor para incrementar productividad y eficiencia a través de pérdidas sociales a nivel global.

\section{- Anonimización}

La búsqueda de instrumentos opacos para volverse anónimos se convierte en un potencial riesgo para la estabilidad financiera. El secreto financiero permite toda clase de actos ilegales e ilícitos. Una de las soluciones propuestas por el GAFI es que los países mantengan registros de "beneficiarios finales" de personas jurídicas y fideicomisos (Knobel, 2017) lo que permitiría detectar actividades delictivas, así como prácticas tributarias agresivas y la posibilidad de usar la IED como forma de esconder capitales locales para beneficiarse de incentivos fiscales.

\section{- Estabilidad financiera}

Pensar que al cerrar los centros financieros offshore se resuelve el tema de los FFl es desconocer lo que está pasando en las finanzas internacionales. Los derivados financieros en mercados extrabursátiles representan un riesgo latente para la estabilidad financiera.

Instrumentos financieros que se suponía iban a dispersar el riesgo pero que en realidad lo intensificaron al convertir la volatilidad de las operaciones a corto plazo en un terreno propio para rápidas ganancias especulativas (Harvey, 2014, p. 236).

Estos instrumentos que fomentan la creación de grandes volúmenes para mantener los stocks utilizan modelos matemáticos que dan la apariencia de estar bajo control cuando al operar por fuera del mercado, tienen efectos macroeconómicos impredecibles como se observó en la crisis del 2008.

Para de Maillard (2011, p. 21) los Estados han dejado que las finanzas sean el árbitro de la economía y la criminalidad se ha infiltrado en el mundo de las finanzas. Desde el punto de vista de la economía neoclásica, los capitalistas establecen estrategias de planificación tributaria y optimización financiera tomando en cuenta las probabilidades de ser capturados por parte de las entidades de control. Esta estrategia depende del grado de aversión al riesgo que tiene cada agente, así como de la posible sanción 
en caso de ser capturado. Para ello, se supone que la sanción juega un efecto disuasivo, así como la crisis reputacional de conocerse públicamente su comportamiento, la competencia con otros actores y las capacidades regulatorias de los entes de control. Estos mecanismos se han mostrado irreales en el caso del banco $\mathrm{HSBC}^{4}$ que ha sido declarado culpable de lavado de dinero en México y ninguno de sus directivos ha sido sancionado penalmente (Senate \& Permanent Subcommittee, 2012). En Estados Unidos, se aplica el concepto de enjuiciamiento diferido a empresas y se habla de cómo los bancos son muy grandes para ir a la cárcel (Radden Keefe, 2017).

\section{- Autorregulación y atomización de la agenda reguladora}

Como lo menciona Ugarteche, el principio de las regulaciones Basilea es que "la autorregulación funciona. Sin embargo, la evidencia bancaria de 2008 en Estados Unidos, Gran Bretaña, Alemania, Suiza e Islandia, por nombrar los ca- sos más relevantes, demuestra que no lo hace" (Ugarteche, 2016, p. 132). Además, las regulaciones actuales de Basilea II y III no se están implementando en la región (King, 2018).

Tanto los centros financieros offshore como los derivados financieros están fuera del ámbito de regulación de Basilea, lo cual no deja de ser inquietante por sus efectos previos. Además, la regulación es simbólica: Basilea beneficia a los grandes bancos en detrimento de los pequeños (Lall, 2012)

La institucionalidad de regulación financiera se encuentra atomizada en seguros, valores, seguros de depósito, bancos y lavado de dinero lo que no permite mirar la integralidad de las actividades que facilitan los FFI.

Las instituciones financieras utilizan también las puertas giratorias a su favor para cooptar a los mejores funcionarios que trabajan en los entes regulatorios y ejercen el lobby para influir en la aplicación de las regulaciones.

\footnotetext{
${ }^{4}$ Banco británico cuyas siglas quieren decir "The Hong Kong and Shanghai Banking Corporation".
} 


\section{CONCLUSIONES}

Las EMN y los bancos son actores principales que gozan de privilegios políticos y económicos y definen los intereses de los FFI. El dumping social es sin duda un elemento perverso de la actual globalización que genera ganancias de productividad y eficiencia en las empresas con costos sociales globales.

Las finanzas son un tema polémico, presentan grandes riesgos con respecto a los derivados financieros, así como por el hecho de estar a su libre albedrío y, además, tienen repercusiones económicas mundiales como se observó en la crisis del 2008. La estabilidad financiera global es un punto clave y necesario porque ante su falta son los más débiles quienes la sufren. Estas contradicciones son propias del capitalismo y están permeadas por el interés pecuniario y por la lógica de corto plazo que recurre a cualquier medio para ser ilusoriamente competitivo con poderes estructurales a su favor.

Respondiendo a la pregunta formulada se puede concluir que los FFI son un problema regional por la asimetría de poder que existe y ha existido históricamente entre países centrales y periféricos. Desde puntos tan sencillos como la definición del objeto de estudio hasta el reciclaje financiero pasando por temas de representación en la toma de decisiones, hay sin duda un poder estructural que normaliza las diferencias.

Se requieren propuestas propias por parte de los países periféricos y esto no quiere decir que no existan puntos de acuerdo sobre los que se pueda discutir e impulsar alternativas en acuerdos internacionales más incluyentes. 


\section{REFERENCIAS BIBLIOGRÁFICAS}

Bhaduri, A. (2011). Repensar la economía política. Buenos Aires: Manantial. de Maillard, J. (2011). L'arnaque - La finance au-dessus des lois et des règles. France: Gallimard.

Frieden, J. A., \& Lake, D. A. (2003). International Political Economy: Perspectives on Global Power and Wealth (4th ed.). London: Routledge.

García-Bernardo, J., Fichtner, J., Takes, F. W., \& Heemskerk, E. M. (2017). Uncovering Offshore Financial Centers: Conduits and Sinks in the Global Corporate Ownership Network. Scientific Reports, 7(1). https://doi.org/10.1038/s41598017-06322-9

Gilpin, R. (2001). Global Political Economy Understanding the international economic order. New Jersey: Princeton University Press.

Harvey, D. (2014). 17 contradicciones y el fin del capitalismo. Quito: IAEN.

Hong, Q., \& Smart, M. (2010). In praise of tax havens: International tax planning and foreign direct investment. European Economic Review, 54(1), 82-95. https:// doi.org/10.1016/j.euroecorev.2009.06.006

King, K. (2016). Scopy Study on International Arquitecture. Quito: Latindadd.
King, K. (2018). Economía política de los flujos financieros ilícitos en la economía mundial. Universidad de Sevilla, Sevilla.

Knobel, A. (2017). Regulación sobre beneficiarios finales en América Latina y el Caribe. Inter-American Development Bank. https://doi. org/10.18235/0000911

Lake, D. A. (2008, junio 19). International political economy A maturing interdiscipline. En B. Weingast \& D. Wittman (Eds.), The Oxford Handbook of Political Economy. New York: Oxford University Press.

Lall, R. (2012). From failure to failure: The politics of international banking regulation. Review of International Political Economy, 19(4), 609. https://doi.org/10.1080/0969229 0.2011 .603669

Lenin, V. I. (1939). The export of capital. En Crane, George \& Amawi, Abla (Eds.), The Theoretical Evolution of International Political Economy (second). New York: Oxford University Press.

Prebisch, R. (1987). Capitalismo periférico: crisis y transformación. México: Fondo de Cultura Económica.

Radden Keefe, P. (2017, julio 31). Limited Liability Why Corrupt Bankers Avoid Jail. The New Yorker. 
Recuperado a partir de http:// www.newyorker.com/magazine/2017/07/31/why-corruptbankers-avoid-jail

Senate, U., \& Permanent Subcommittee, on I. (2012). U.S. Vulnerabilities to Money Laundering, Drugs, and Terrorist Financing: HSBC Case History. Washington, DC: US Senate. Recuperado a partir de https://www. hsgac.senate.gov/imo/media/ doc/PSI\%20REPORT-HSBC\%20 CASE\%20HISTORY\%20(9.6).pdf

Strange, S. (1986). Casino capitalism. Oxford: Basil Blackwell.
Strange, S. (1994). State and markets (2nd ed.). London: Continuum.

Strange, S. (2002). Finance in Politics: An Epilogue to Mad Money.

Tax Justice Network. (2015). Capital Flight, Illicit Flows - Tax Justice Network. Recuperado 21 de marzo de 2017, a partir de http:// www.taxjustice.net/topics/inequality-democracy/capital-flight-illicit-flows/

Ugarteche, O. (2016). Historia crítica del Fondo Monetario Internacional. México: Capital Intelectual. 